\title{
Face Recognition Based on Attendance Management System
}

\author{
Rajeev Yadav, Sumit Chauhan, Meenu, Swati Gupta
}

\begin{abstract}
Attendance is very important task for an organization like school, colleges, companies etc. Taking of attendance manually is very time consuming. It takes up to 5 to 20 minutes and it depends upon the number of students in a class. Now a day's technology become more advance, so we have to use biometric system for attendance because it takes very less time as compared to taking attendance manually. In this project we are going to make an attendance system using face recognition. There are many algorithms by which we can make an attendance system using face recognition. In this project we use Viola-Jones Algorithm and Local Binary Pattern Histogram (LBPH) Algorithm. Viola-Jones algorithm is used for face detection and Local Binary Pattern Histogram is used for samples training and face recognition
\end{abstract}

KEYWORDS- Viola-Jones Algorithm, Linear Binary Patterns Histograms (LBPH), Python, Face Detection, Face Recognition

\section{INTRODUCTION}

We can take attendance manually and with the help biometric system. Taking attendance manually is difficult for teachers and students also. Many problems are arises due to taking attendance manually like proxy mark by students, this process is time consuming and sometimes, the paper on which we give our attendance is lost. Taking attendance using biometric is very less time consuming, there is no chances of proxy and there is no chance of paper lost. In this project we are going to learn how we take attendance using our face. Taking attendance using our face we have to learn about some algorithms.

Manuscript received May 15, 2020

Rajeev Yadav, Department of Computer Science, Amity University, Gurugram, Haryana, India

Sumit Chauhan, Department of Computer Science, Amity University, Gurugram, Haryana, India

Meenu, Assistant Professor, Department of Computer Science, Amity University, Gurugram, Haryana, India (email: mvijarania@ggn.amity.edu)

Swati Gupta, Assistant Professor, Department of Computer Science, Amity University, Gurugram, Haryana, India.
There are many algorithms available for this process [1] like Viola Jones Algorithm, Ada-Boost Algorithm, etc are used for face detection and Linear binary patterns histogram, Eigenfaces algorithms, etc, are used for face recognition. But in this, project we are going to use Viola-Jones for face detection and Linear binary patterns histogram Algorithm for face recognition. We will also create a database in which in we have to capture the images for training the algorithm and we have captured the images of a student with same ID. For implementation of this project, we are going to use python because it is simple and easy to use. There are fields in which we have to develop the facial algorithms like criminal identification, police authentication, defence services, bank services, online payments etc. But in this project we learn to make face recognition based attendance management system.

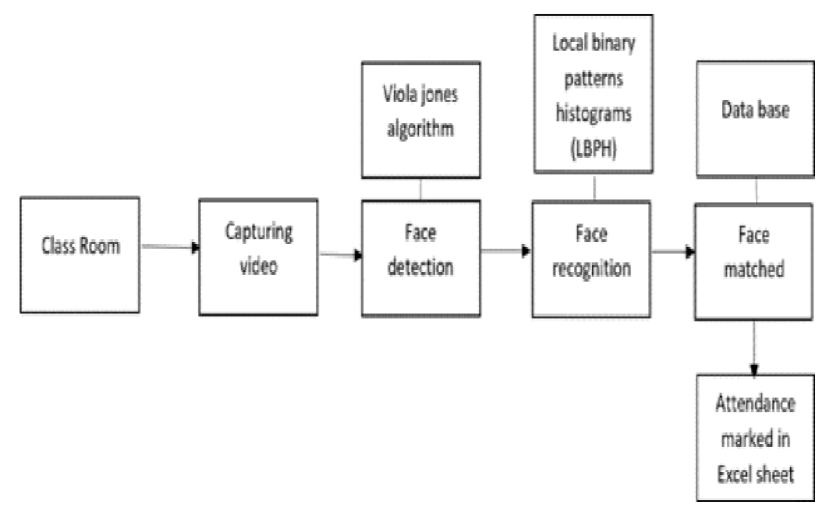

Fig 1: Block diagram

\section{LITERATURE REVIEW}

Ms. Varsha Gupta et al. [2] proposed that there are different face detection techniques. Basic patterns for face detection are nose, eye, ears. Face detection is detecting face from an image or a video. There are different types of detection techniques, each have advantage and disadvantage discussed in paper.

Sudhir Bussa et al. [3] they used the open CV-based facial recognition approach. This model integrates a camera that captures a Viola Jones face detection algorithm, an Ada-Boost algorithm etc. input image, an algorithm to detect the face from an input image, encode and identify the face, marking the presence in a spreadsheet. The training database is created by training the system with the faces of 


\section{Face Recognition Based On Attendance Management System}

authorized students. The cropped images are then archived as a database with their respective labels. The functionality is extracted using the LBPH algorithm.

In this paper face recognition for human faces [4] are employed through Viola Jones algorithm for face identification LBPH in favor of feature extraction, Euclidean distance classifier is for face recognition. In general the steps employed to achieve this are the following: creating a dataset, face acquisition, feature extraction, and finally classification. The entire work is done in opencv using python.

Abin Abraham et al. [5] proposed the various factors or methods to take attendance depending on the number of persons, departments etc. There are various methods for taking attendance like calling by roll number, swiping the card, etc depending on the no. of persons. In some place where no. of employees is in greater number they use biometrics like iris, handprint, finger print etc. Now as technology is growing day by day the methods are also developing for attendance, so here we are working on a system that automatically take attendance using face recognition.

\section{METHODOLOGY}

\section{A. Face Detection}

Face detection is a process of capturing a face from an image and a video. There are so many algorithms available for face detection like Viola-Jones Algorithm, Ada-Boost Algorithm etc. In this project we are using Viola-Jones Algorithm for face detection.

Now we are going to discuss how Viola Jones algorithm:-

Viola-Jones [6] made for front faces so it can detect best foresight instead of looking up or down before tracing, faces of the image is changed to gray-scale because it is easier to work with it and less data is also available to process the faces of grayscale image. Viola-Jones ones has a search outline for a face within a box. it is essentially looking for features such as these haar like features. In this case it uses a bigger box size and takes bigger steps to show. In general you could change the box size and step size as per your requirements. There are several boxes designed to identify small steps. Features like the face and haar-like features. All cases have data that's been packed together, helping the algorithm to determine the position of face.

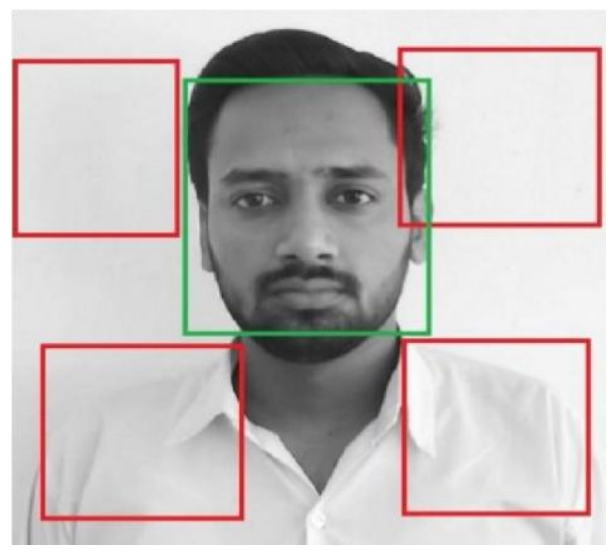

Fig 2: Face detection
This algorithm has four steps:

1. Haar-like features

2. Creating an Integral Image

3. Adaboost Training

4. Cascading Classifiers

\section{Haar-like features}

Now, the pictures below depict two sides black side and white side, these are the features shown by machine. Meanwhile at some instance one of the sides is shown lighter as edge of an eyebrow, while sometimes it is shinier in the middle which is depicted as nose. Viola-Jones identified three Haar-like features.in the research and these features are Line features, Edge features and four-side features.

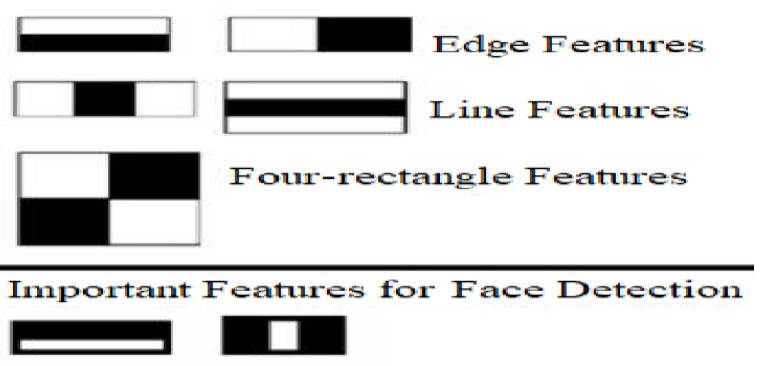

Fig 3: Haar-like features

$\rightarrow$ Implementation of haar like feature on image

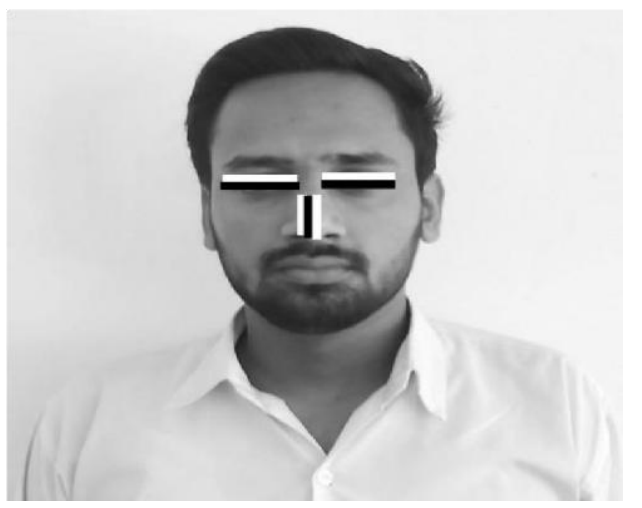

Fig 4: Haar- like features on Image

\section{Creating an integral image}

In this step [7], an input image is converted to an integral image as shown in the figure. The integral image at location $(\mathrm{x}, \mathrm{y})$ contains the sum of the pixels to the left and above (x, $\mathrm{y})$.

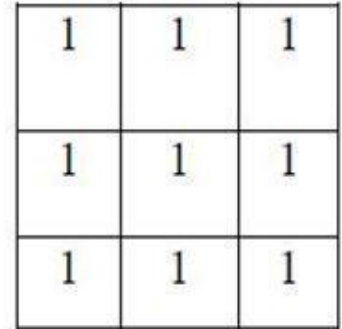

$\mathrm{S}$

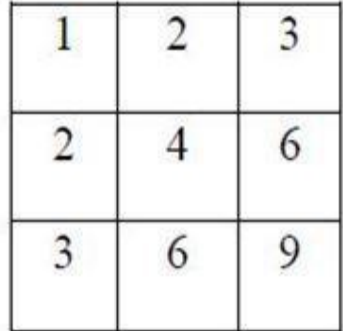

(b) (a)

Fig 4: (a) input image and (b) integral image 
This calculates the sum of the pixels within any specified rectangle using only four values. In the full image, these values are the pixels that look like the edges of the rectangle in the input image.

\section{Adaboost Training}

This algorithm acquires an image of 24 by 24 pixels. The possible output characteristics of the image are one hundred eighty thousand. There will be a lot of data extracted from the image. it is practically impossible to do this real time. For adaptive reinforcement to be used for the realization, strong filing cabinet and reduces calculation time. The classifier will add up the characteristics and theirs weights of images. The classifier will take important facial features and stored in the filing cabinet that makes the stronger classifiers.

\section{Cascading Classifiers}

It is used to increase the speed and precision of our model. [8]In cascade, each phase is composed of a strong classifier. So all the features are grouped into multiple stages where each stage has a number of features. So, let's start by taking a secondary window and inside this secondary window, let's take our best functionality and see if it is present in the image inside the secondary window. If it's not in the secondary window, we don't even look at the secondary window, we just discard it. So if it is present, let's examine the second function in the secondary window. If it is not present, we reject the secondary window. We continue because of the number of functions we have and we reject secondary windows without the function. Evaluations can take a split second, but since you have to do it for each function, it can take a long time. Cascading greatly accelerates this process and the machine can deliver results much faster.

$$
F(x)=a 1 f 1(x)+a 2 f 2(x)+a 3 f 3(x) \ldots \ldots
$$

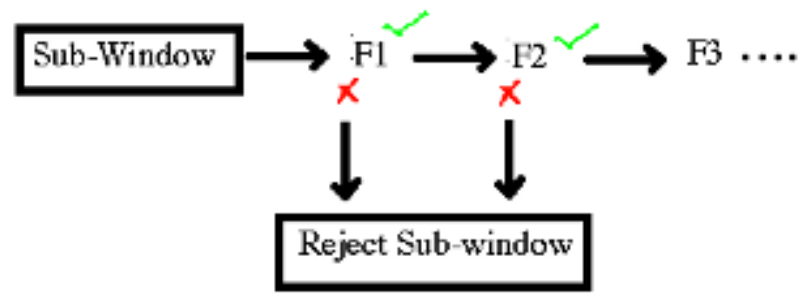

Fig 5: Cascading

\section{B. Image Training}

After face detection we have to train the images with the help of LBPH algorithm.

Local Binary Patterns Histograms (LBPH) [9] uses for parameters.

- Grid X: means cells in the horizontal direction. It is generally set to 8 .
- Grid Y: means cells in the vertical direction. It is generally set to 8 .

- Radius: circular binary pattern is build using radius. It is generally set to 1 .

- Neighbours: these are the sample points to build circular local binary pattern. The more sample points higher the computational cost. It is generally set to 8 .

\section{Training the Algorithm}

It is an important step for face recognition. We have to use an images dataset to train the algorithm. While training keep in mind that we need to set an ID for each image, and we have to take minimum 40 image of each person. Higher the number of images, higher we get the accuracy. Images of the person must have the same ID. We could also set any text (like name, age etc) with image so that when we the recognize the image it will text about that image.

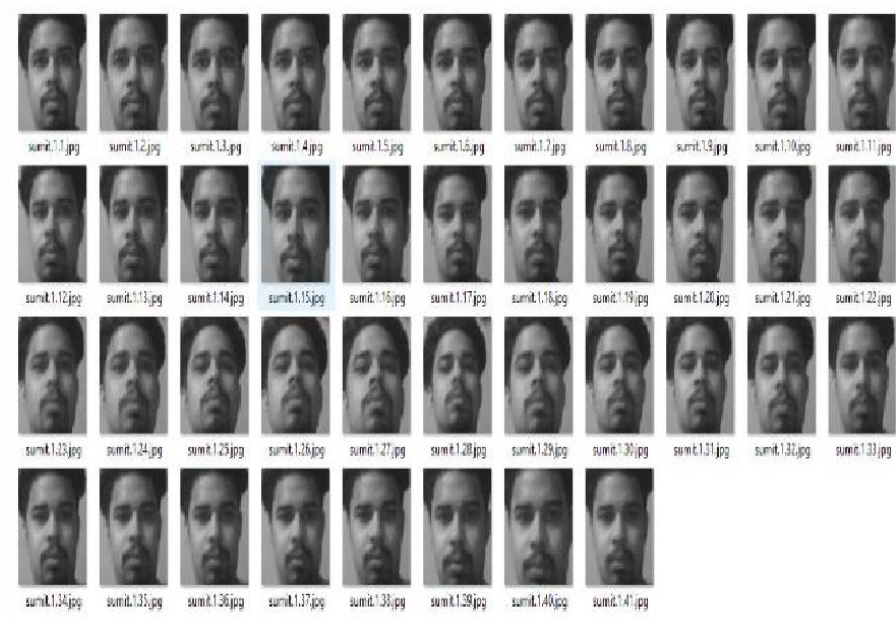

Fig 6: Faces dataset for training the algorithm

\section{Applying the LBP operation}

First, we have to create an image that describe the original image in better way. For this algorithm uses sliding window [10] which based on the radius and neighbors parameters. Now we are going to how we can apply the LBH operation

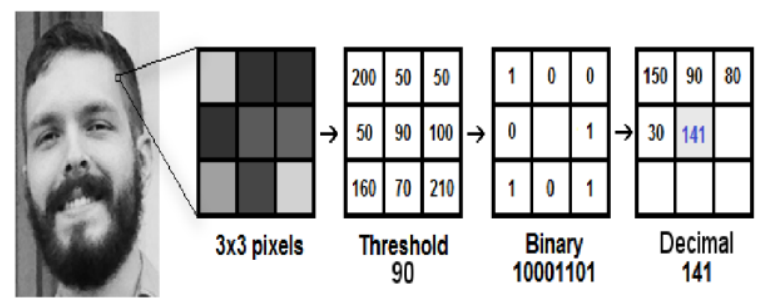

Fig 7: Image unit point converted into pixel

Keep in mind that the image must be in greyscale for better results and then takes a unit point from that image and assume that unit point is in widow of 3 by 3 pixels. It can be represented as $3 * 3$ matrix having the intensity of each pixel. Consider the central value of matrix used as threshold and rest values are the neighbors values and then each neighbour set as binary value. Set 1 for equal and 


\section{Face Recognition Based On Attendance Management System}

higher values and 0 for smaller values. Now the matrix has only binary values and then concatenates each binary value. After that convert binary value in decimal value and then set it to central value of the matrix. That is actually a pixel from the original image and this will represents the better characteristics of the original image.

\section{Extracting the Histograms}

In this we have to use the image, [11]which we got from training and divide that image into multiple grids using the parameters gird $\mathrm{x}$ and grid $\mathrm{y}$. Using the image below, we must extract the histogram of each region and each histogram contains only 256 positions representing the occurrences of each pixel intensity and therefore we must concatenate each histogram to create a larger histogram. The original image comes from the final histogram.
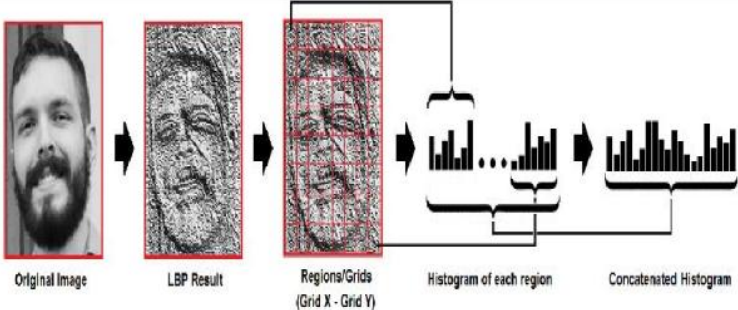

Fig 8: Division of the image into multiple grids

\section{Performing the face recognition}

After training the images it is easy to recognise the face from an image and a video. Each histogram is created used to represent each image from the training set.

Suppose we have to find an input image from given dataset, to do so[12] we have to compare two histograms and then return the image with closet histogram and we have different methods to compare the histogram like Euclidean distance, chi, absolute value, etc but we use Euclidean distance. At last we got the output image with ID.

$$
D=\sqrt{\sum_{i=1}^{n}\left(\text { hist } 1_{i}-\text { hist } 2_{i}\right)^{2}}
$$

Fig 9: Formula for compare two histograms

\section{Face Recognition}

Face recognition is a process in which we have identified or verified the identity of an individual using their face. After face detection and image training we can easily recognize the face of a person.

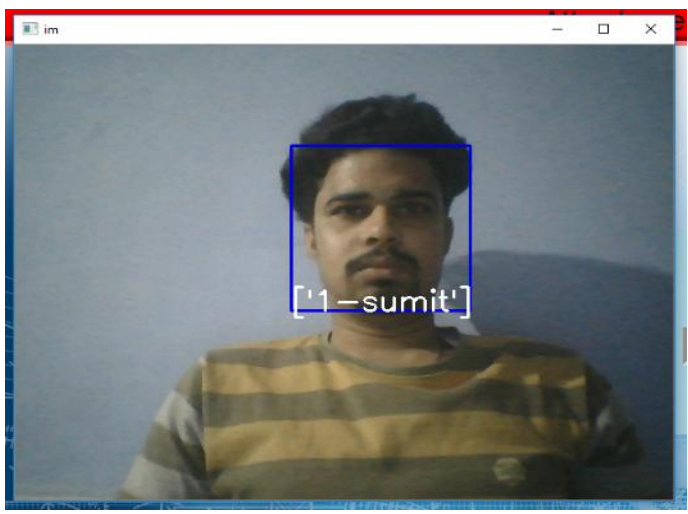

Fig 10: Recognizing the face

\section{RESULT AND DISCUSSION}

The main working of this project is to make face recognition based on attendance management system that is used in an organisation (school, collage, companies, etc) to capture the face images and mark the attendance of the students in the excel sheet with ID and Name. The algorithm gives the result when the given image is perfectly matched with the database faces. In this project we are taking 40 images each person for better accuracy to perform the experiment, the attendance is marked in the excel sheet automatically.

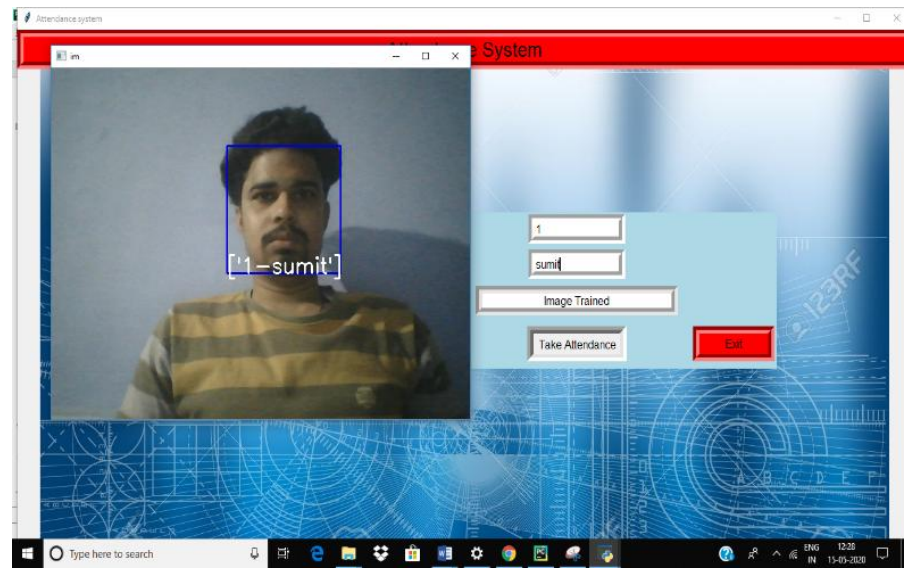

Fig 11: Shows the output of this experiment.

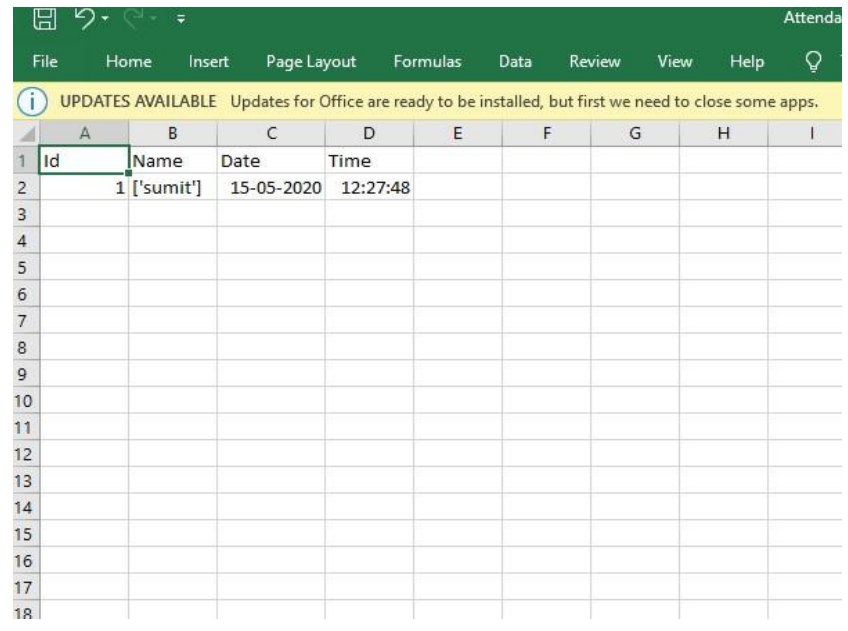

Fig 12: Attendance marked in the excel sheet 


\section{CONCLUSION AND FUTURE SCOPE}

In this project face detection is carried out by using voila jones dace detection algorithm feature extraction by using Local Binary Patterns Histograms and classification by using Euclidean distance classifier. The proposed system is implemented using OpenCV and PyCharm. From the above implementation I can conclude that LBPH and Euclidean distance has better recognition rate. On the base of analysis carried out in the present examine, the following suggestions are accessible for further research and it is valuable to concentrate on the following issues in future work. The proposed system is modified to support in all the conditions such as brightness, wearing goggles, beard, in case of twins by minimum distance classifier. The proposed method is tailored in the evolution of genetic properties for facial expressions is studied for different security metrics is helpful in securing government confidential databases and criminal detection

\section{REFERENCES}

[1] V. K. Polamarasetty, M. Reddy Reddem, Dheeraj Ravi, M. Sai Madala "Attendance System based on Face Recognition “, vol. 5, no. 2395-0072, 2018

[2] Varsha Gupta and Dipesh Sharma, "A Study of Various Face Detection Methods", vol. 3, no. 2319-5940, 2014

[3] S. Bussa, S. Bharuka, A. Mani and S. Kaushik, "Smart Attendance System using OpenCV on Facial Recognition", vol. 9, no. 2278-0181, 2020

[4] Ms. Priyanka Dhoke, Mr. M.P. Parsai, "MATLAB based Face Recognition using PCA with Back Propagation Neural Network”, 2014.

[5] Abraham, M. Bapse, Y. Kalaria and A. Usmani, "Face Recognition Based Attendance System", vol.22, no.2278-0661,2020

[6] Rohan Gupta(2019), "Breaking Down Facial Recognition: The Viola-Jones Algorithm", accessed on 5May, 2020

https://towardsdatascience.com/the-intuition-behind-f acial-detection-the-viola-jones-algorithm-29d9106b69 99>

[7] Ms. Suma S L, Mr. S. Raga, "Real Time Face Recognition of Human Faces by using LBPH and Viola Jones Algorithm” Vol.6, no. 2320-7639, 2018

[8] Mr. Rajath S Bharadwaj, Mr. Tejus S Rao, Mr. Vinay T $\mathrm{R}$ "Attendance Management Using Facial Recognition”,, vol. 8, no.2278-3075, 2019

[9] Mr. Kelvin Salton do Prado(2017), "Face Recognition: Understanding LBPH Algorithm", accessed on 5May, 2020"

https://towardsdatascience.com/face-recognitionhow-lbph-works-90ec258c3d6b

[10] Mr. P. Viola and Mr. M. Jones "Rapid object detection using a boosted cascade of simple features.", vol. 1, 2001.
[11] Mr. J. Joseph and Mr. K. P. Zacharia, “An Automatic Attendance Management System Using Face Recognition," vol. 2, no.327-330, 2013.

[12] Ms. Rekha AL and Dr. Chethan H K, “An Automated attendance system using face recognition through videosurveillance", vol. 1, no.1327-1330, 2014. 